\title{
Energy Efficient LEACH
}

\author{
M. M. Chandane \\ Department of Computer \\ Technology, VJTI, Mumbai, India
}

\author{
Ashish Mohta \\ Department of Computer \\ Technology, VJTI, Mumbai, India
}

\author{
S. G. Bhirud \\ Department of Computer \\ Technology, VJTI, Mumbai, India
}

\begin{abstract}
Sensors are battery operated devices and are normally placed in the region where humans could not reach easily. Therefore these devices need to be used carefully so as to extend the network life. Network layer plays important role for transmission of data from source node to destination node. Considerable amount of energy is consumed for routing the data between the source and destination node. Hence, to prolong the lifetime of sensor network, it is essential to think for routing protocol that takes care of energy consumption during data transmission process. In this paper a new protocol called Advanced LEACH (ALEACH) is suggested, which is a modified version of LEACH protocol. The objective of this protocol is to develop more energy efficient routing protocol so as to increase the overall life of Sensor Network. ALEACH protocol selects the cluster head randomly from the set of devices whose residual energy is greater than the average energy of the network. We have implemented and tested ALEACH in NS2 and results are compared with LEACH protocol for various parameters. The obtained result shows that ALEACH performs better than its counterpart.
\end{abstract}

\section{Keywords}

Clustering, LEACH, ALEACH, Time Division Multiple Access (TDMA), Ad hoc networks, SCADA systems.

\section{INTRODUCTION}

Wireless Sensor Network (WSN) is a new computing paradigm that emerged from the fusion of the supervisory control and data acquisition (SCADA) systems and Ad hoc networks technologies [1][2]. WSN derives the networking characteristics of ad-hoc network and combines it with the hardware facilities of tiny wireless sensors. Once a sufficient number of nodes have been deployed, the sensor network can be used to fulfill its task, such as measuring the physical phenomena. This task can be issued and supervised by an external entity connected to the WSN, such as manager or supervisor.

WSNs are increasingly attractive tools to detect, monitor and control environmental conditions. WSNs can be used to bridge the gap between the physical and the virtual world. The distributed wireless sensing has variety of applications such as medical, home security, machine diagnosis, military Information applications, environmental monitoring, agriculture, etc. Fig 1 shows the working concept of WSN.

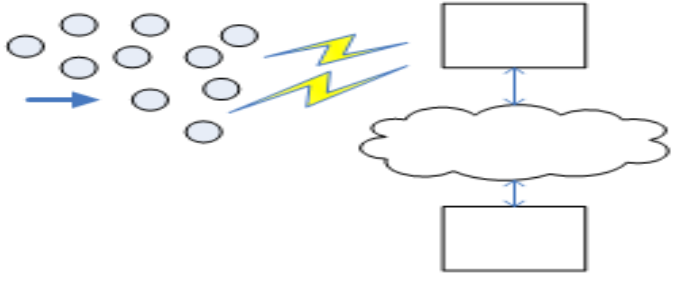

Fig 1: Working of WSN
Routing is used to make any network more useful and efficient. For WSN main aim of any routing protocol is to route packets to the destination and save the sensor energy for transmission [3]. Low energy adaptive cluster head (LEACH) is perhaps the first cluster based routing protocol for wireless sensor networks, which uses a stochastic model for cluster head selection [4]. LEACH has motivated the design of several other protocols [4] [5]. Like other routing protocols LEACH is also having some limitations like:

- Cluster head $(\mathrm{CH})$ selection is random and it does not take node's energy into consideration.

- The selected $\mathrm{CH}$ may die during data transmission and cause the data loss.

Our proposed protocol ALEACH deals with these problems by considering the residual energy of the nodes for the selection of cluster head at the time of cluster head selection process. ALEACH is implemented in NS2 and obtained results are compared with LEACH.

This paper is organized as follows: Section II presents the related work. Proposed algorithm is given in section III. Section IV presents the implementation and environmental set-up. Simulation parameters and the results are discussed in section $\mathrm{V}$ followed by conclusion and references.

\section{RELATED WORK}

In a cluster based routing protocol, sensor nodes are partitioned into many groups called clusters, which integrates data collected from member sensor nodes and transmits them to the sink node of the network. Each of the clusters in this network, has a cluster head which collects data from sensor nodes within its group, completes data aggregation, and sends them to the sink node of the network. Such data aggregation can reduce the consumption of node energy and the transmission delay as compared to multi-hop routing protocols [6].

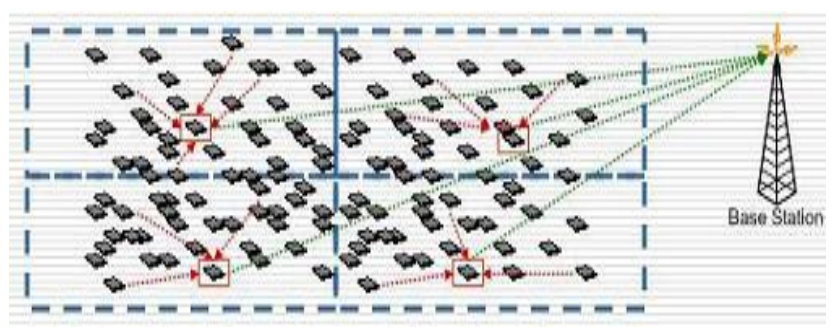

Fig 2: Clustering principle [6]

Advantages to a clustering network are the reduction of an overhead of route establishment, minimization of the size of the routing table, and stabilization of the network topology. The clustering network can make resource management, bandwidth allocation more efficient and also does node positioning and management of transmitting power possible. 
In addition, the clustering scheme in a wireless sensor network enables an aggregate data of cluster member nodes at the cluster head and can easily provide the network scalability due to increase in number of nodes. Consequently, in a large sensor network, the clustering based routing algorithm can be a possible approach to maintain network configuration management and to make data aggregation. In this approach, all nodes in a network can become a cluster head but must belong to only one cluster. The algorithm should minimize the overhead of clustering setup messages and establishing times. Additionally, the algorithm must maintain a stable network configuration, routing, network efficiency, with a minimization of energy consumption [6]. Many clustering algorithms have been proposed, most of which are based upon node identifier, node connectivity, and node weights. Some of better-known cluster based hierarchical routing protocols are LEACH, LEACH-Centralized, and Chain-based level PEGASIS (Power Efficiency Gathering in Sensor Information Systems). Fig 2 shows the working of clustering principle.

\subsection{Leach}

Low Energy Adaptive Clustering Hierarchy (LEACH) is the first hierarchical cluster-based routing protocol for wireless sensor network which partitions the nodes into clusters, in each cluster a dedicated node with extra privileges called Cluster Head $(\mathrm{CH})$ is responsible for creating and manipulating a TDMA (Time division multiple access) schedule and sending aggregated data from nodes to the base station (BS) Remaining nodes are cluster members [3]. Working of LEACH is shown in fig 3 and its phases are shown in fig 4 . The phases of LEACH consists of following activities.

\section{- $\quad$ Set-up Phase}

\section{○ Advertisement Phase \\ ○ Cluster Set-up Phase}

- Steady state Phase
- Schedule Creation
- Data Transmission

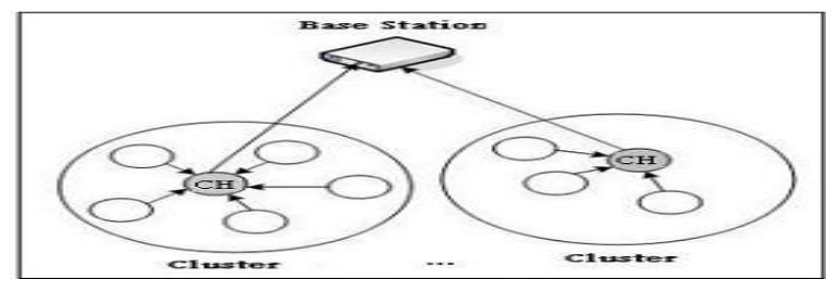

Fig 3: Working of LEACH Protocol [6]

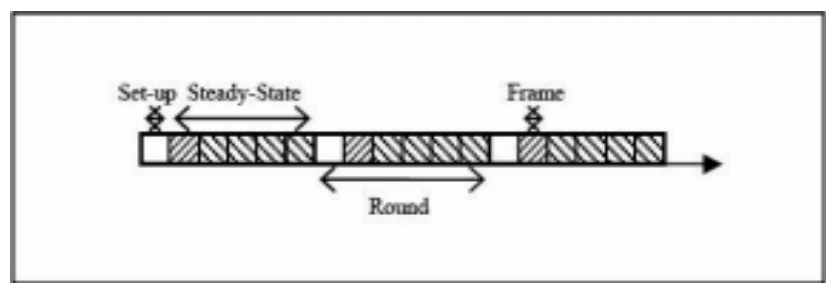

Fig 4: Phases of LEACH protocol

\subsubsection{Setup Phase}

During this phase, each node decides independent of other nodes if it will become a $\mathrm{CH}$ or not. This decision takes into account, when the node served as a $\mathrm{CH}$ for the last time (the node that hasn't been a $\mathrm{CH}$ for long time is more likely to elect itself than nodes that have been a $\mathrm{CH}$ recently).

In the following advertisement phase, the $\mathrm{CHs}$ inform their neighborhood with an advertisement packet that they become $\mathrm{CHs}$. Non- $\mathrm{CH}$ nodes pick the advertisement packet with the strongest received signal strength.

In the next cluster setup phase, the member nodes inform the $\mathrm{CH}$ that they become a member to that cluster with "join packet" contains their IDs using CSMA. After the clustersetup sub phase, the $\mathrm{CH}$ knows the number of member nodes and their IDs.

\subsubsection{Steady-state phase}

Schedule creation: Based on all messages received within the cluster, the $\mathrm{CH}$ creates a TDMA schedule, pick a CSMA code randomly, and broadcast the TDMA table to cluster members.

Data transmission: Nodes send their data during their allocated TDMA slot to the $\mathrm{CH}$. This transmission uses a minimal amount of energy (chosen based on the received strength of the $\mathrm{CH}$ advertisement). The radio of each non- $\mathrm{CH}$ node can be turned off until the nodes allocated TDMA slot, thus minimizing energy dissipation of these nodes. When all the data has been received, the $\mathrm{CH}$ aggregates these data and sends it to the BS. This aggregation of data at each $\mathrm{CH}$ leads to reduction in amount of data that is to be transmitted to the base station.

Although LEACH protocol acts in a good manner, it suffers from many drawbacks like;

- $\quad \mathrm{CH}$ selection is random, that does not take energy consumption into account.

- Sensors die steeply after some point or time and due to this sudden death of sensors large data loss occurs.

- Energy consumption is also high in these networks due to low network lifetime and data loss.

To overcome the drawback of LEACH, several researchers are working on it. Here we are discussing some of the variants suggested by various researchers.

\subsection{Leach-C}

This is an extension of the Leach protocol. It uses centralized approach for the formation of the clusters and cluster heads [6].The algorithm execution starts from the base station where the base station first receives all the information about each node regarding their location and energy level and then it runs the algorithm for the formation of cluster heads and clusters. In this protocol also, the number of cluster heads are limited and the process of selection of the cluster head is also random but the base station ensures that the node with comparatively lesser energy does not become a cluster head. The problem with the Leach-C is that it is not feasible for larger networks because the nodes which are far away from the base station will have difficulty in sending their status to the base station and since the role of cluster heads rotates, therefore the distant nodes would not reach the base station in time. This results in increase of communication latency and also delay amplifies.

The routing algorithm of Leach-C is based on two phases, the setup phase and the steady phase. In the set up phase cluster head are selected. The steady phase is the data transmission phase. 


\subsection{Leach-F}

Leach-F [Balakrishnan, Chandrakasan and Heinzelman, 2002] uses the ideas that if the clusters remain fixed and only rotate the cluster head role within the cluster this will save masses of energy and increase the system throughput as well, whereas the disadvantage is that lack of scalability within the network which means new nodes cannot be added.

\section{PROPOSED ROUTING PROTOCOL}

The proposed ALEACH protocol considers only those nodes for the selection of cluster head, whose residual energy is more than the average energy of the network as against the random selection of cluster head from the set of sensor nodes used by the LEACH. ALEACH employs the same clustering approach as LEACH during initial phases. Adjacent nodes are organized into clusters with one node from each cluster acting as the cluster head. ALEACH extends LEACH by applying the check for residual energy of node before making it cluster head, the energy should be more than or equal to the average energy of the network, so that the node will not die during the transmission process. The average energy is calculated as follows:

Etotal $=\sum \mathrm{E}(\mathrm{Ni})$

Eavg $=$ Etotal $\div \mathrm{nn}$

Where

Etotal is the total Energy of the network

Eavg is the average Energy of the network

$\mathrm{nn}$ is the total number of nodes

- The choice of the cluster head $(\mathrm{CH})$ is determined by the amount of remaining energy in a node

- The energy should be more than or equal to the average energy of the network

\section{IMPLEMENTATION AND ENVIRONMENTAL SET-UP}

The proposed algorithm has been implemented in NS-2 [7]. Obtained results are compared with LEACH algorithm in terms of network lifetime and number of data packets received at Base Station.

\subsection{Environment Setup}

For this experimentation model, 100 nodes were taken, which were randomly deployed and distributed over $100 \times 100$ square meter area. It is assumed that all nodes are fixed and static in nature since most of the applications of WSN uses static scenario. These simulation models uses the same parameters as used in [5] and are as stated in table 1.

Table 1. Simulation Parameter

\begin{tabular}{|c|c|}
\hline Parameters & Values \\
\hline Network Size & $100 \mathrm{~m} \mathrm{X} \mathrm{100m}$ \\
\hline Location of the Base Station & $(20,175)$ \\
\hline The number of nodes & 100 \\
\hline The number of clusters & 5 \\
\hline The initial energy of nodes & $2 \mathrm{~J}$ \\
\hline Data Packet Length & 512 bytes \\
\hline
\end{tabular}

\section{SIMULATION RESULTS}

Following parameters are used to analyze simulation environment and to compare the results

Network Lifetime: It deals with the life of network. It shows how long the nodes in network are alive over a period of time. Fig 5 shows the lifetime of the network using LEACH and ALEACH protocol. Fig 5 shows that the network with LEACH protocol has lower Network Lifetime than the ALEACH protocol for 100 nodes.

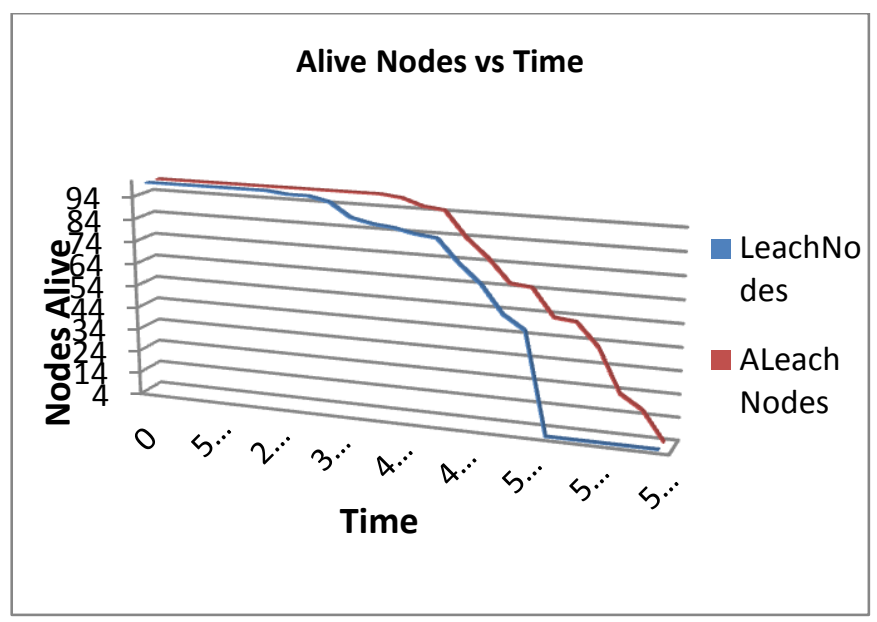

Fig 5: Network lifetime using LEACH and ALEACH

Data Packet Received: Number of data packet received at base station with fixed number of nodes. It shows how effective the protocol is when data transmission is concerned.

Fig 6 shows number of data packets received by the base station for variable number of nodes using LEACH and ALEACH. The performance is tested for varying number of nodes such as 10, 25, 50 and 100 nodes with 2, 3, and 5 cluster heads. Result shows that the network with ALEACH protocol delivers more number of data packets as compared to ALEACH.

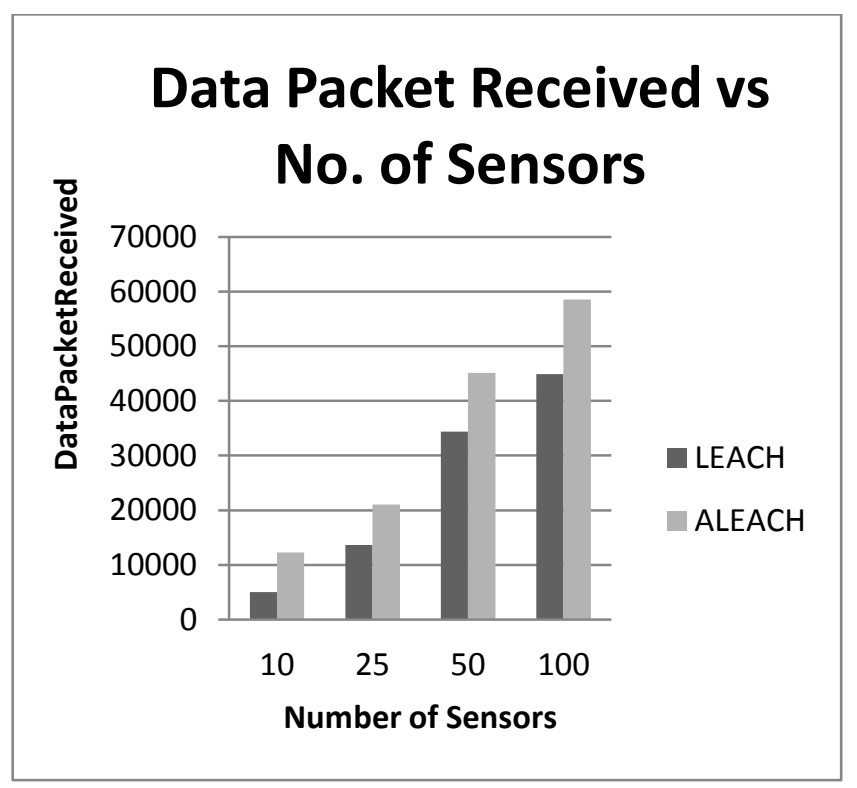

Fig 6: Data Packet Received with different number of Nodes in LEACH and ALEACH

Based on above comparative results it can be concluded that the ALEACH protocol performs much better than LEACH 
protocol as per as network lifetime and delivery of data packets are concerned.

\section{CONCLUSION}

In this work, a new cluster based routing protocol is proposed which is the modified version of LEACH called ALEACH. This protocol considers the residual energy of the nodes as selection criteria for cluster head and also the selected cluster head is ensured that it will not die during the data transmission process. Results of simulation study shows that the proposed protocol provides a longer network lifetime than LEACH. This consideration of residual node energy during cluster head selection process can maintain the balanced energy consumption of the sensor network. Additionally, the protocol test for network scalability shows that ALEACH receives more data packets than LEACH. It is our belief that the proposed protocol can effectively extend the network lifetime without other critical overheads and performance degradation

\section{REFERENCES}

[1] Eiko Yoneki, Jean Bacon, "A Survey of Wireless Sensor Network Technologies: Research Trends and Middleware's Role", University of Cambridge, Sep 2005 .
[2] Hetal Jasani, Kia Makki, and Niki Pissinou, "On Wireless Sensor Networks," Florida International University, Miami, USA, June 2004.

[3] M. Bani Yassein, A. Al-zou'bi, Y. Khamayseh, W. Mardini, "Improvement on LEACH Protocol of Wireless Sensor Network (VLEACH)", International Journal of Digital Content Technology and its Applications Volume 3, Number 2, June 2009.

[4] Heinzelman W., Chandrakasan A., and Balakrishnan H.,"Energy-Efficient Communication Protocol for Wireless Microsensor Networks", 2000.

[5] W. R. Heinzelman, A. Chandrakasan, and H. Balakrishnan, "An Application-Specific Protocol Architecture for Wireless Microsensor Networks," IEEE Transactions on Wireless Communications, Vol. 1, No. 4, pp. 660-670, Oct. 2002.

[6] J. Ibriq and I. mahgoub, "Cluster-Based Routing in Wireless Sensor Networks: Issues and Challenges", Proceedings of the 2004 Symposium on Performance Evaluation of Computer Telecommunication Systems, pp.759-766, Jul. 2004.

[7] Information Sciences Institute, "The Network Simulator ns-2" http://www.isi.edu/nanam/ns/, University of Southern California. 